\title{
Neues Schäumverfahren mit CO, als Treibmittel für PUR-Formteile
}

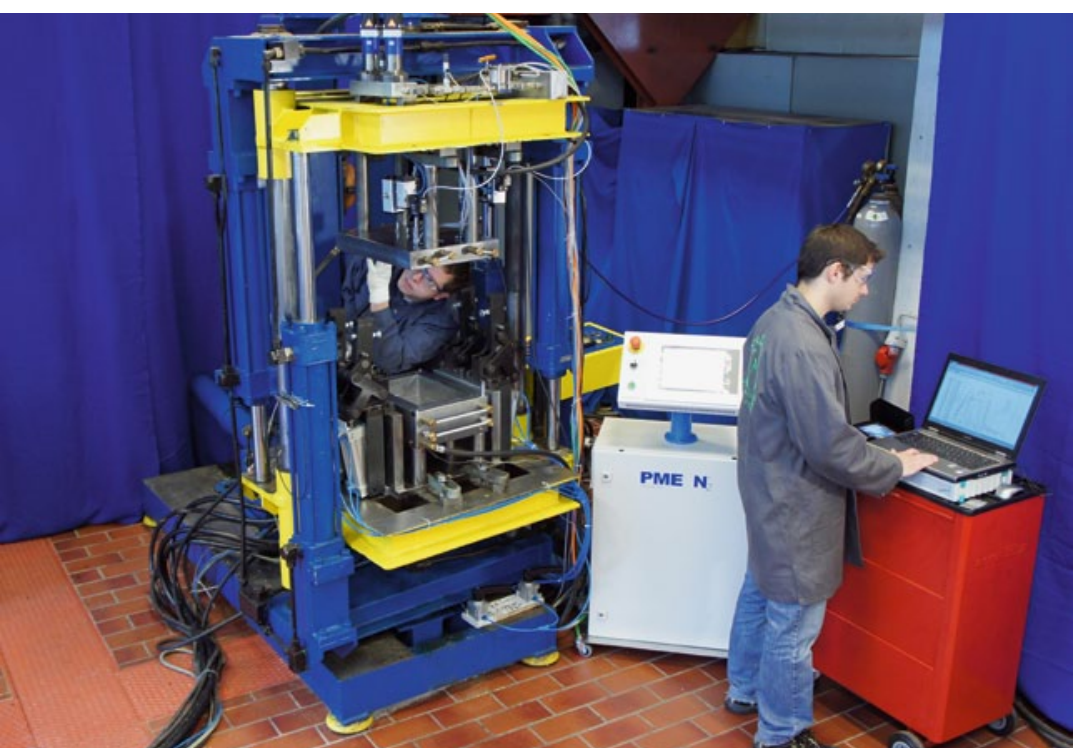

Zur Herstellung von geschäumten Polyurethan-Formteilen entwickelt das Institut für Kunststoffverarbeitung (IKV) an der RWTH Aachen eine neue Schäumtechnik. Sie soll den Einsatz von $\mathrm{CO}_{2}$ als Treibmittel ermöglichen.
Erste Untersuchungen zeigen nach Angaben der Aachener Wissenschaftler, dass das neue $\mathrm{CO}_{2}$-Schäumverfahren deutlich geringere Raumgewichte erzielt als dies bisher möglich war. Um ein kontrolliertes Aufschäumen des Reaktionsgemischs zu erreichen, werde in der Schäumform ein Gasgegendruck erzeugt. Dieser Werkzeuginnendruck, der über dem Dampfdruck des gelösten $\mathrm{CO}_{2}$ liegt, soll ein frühzeitiges Aufschäumen verhindern. Zeitpunkt und Geschwindigkeit des Aufschäumens ließen sich mit einer gezielten Reduktion des Innen- drucks steuern. Auf diese Weise werde ein zu schnelles Aufschäumen und Kollabieren des Schaums unterbunden.

Die IKV-Forscher entwickelten für das neue Verfahren gemeinsam mit dem Ettenheimer Unternehmen PME Fluidtec ein abgedichtetes Werkzeug mit Ventiltechnik sowie eine entsprechende Prozesssteuerung. Darüber hinaus wurde die Hochdruck-Dosiermaschine HS500 der Firma Hennecke an die neue Prozesstechnik angepasst.

Das IKV hat die neue Technik bereits mit Erfolg in Betrieb genommen, heißt es. Bereits die Vorversuche sollen Weichschaumbauteile mit einer Dichte von rund 90 kg/ $\mathrm{m}^{3}$ geliefert haben. Mit diesem Verfahren könne somit der Bereich der individuell einstellbaren mechanischen Eigenschaften des PUR-Schaums weiter vergrößert werden. Das mit $\mathrm{CO}_{2}$ geschäumte Material lasse sich weicher einstellen als chemisch geschäumtes. Zusätzlich reduziere es den Bedarf an Isocyanat, das für die herkömmliche chemische Schäumreaktion benötigt wird, und trage somit dazu bei, Kosten zu reduzieren.

\section{LEICHTBAUFORSCHUNG UNIVERSITÄT STUTTGART UND DAIMLER KOOPERIEREN}

Um neue Konstruktions-, Simulations- und Verfahrenstechniken im FVK-/CFK-Leichtbau zu erforschen, haben das Institut für Flugzeugbau der Universität Stuttgart und Automobilhersteller Daimler einen Kooperationsvertrag geschlossen. Die langfristig angelegte Kooperation soll die Forschungsaktivitäten der beiden Partner im Forschungscampus Arena-2036 untermauern. „Am Institut für Flugzeugbau werden seit vielen Jahren Faserverbund-Leichtbaustrukturen für die Luftfahrt entwickelt. Unsere Forschungsschwerpunkte Preformtechnologie/ Liquid Composite Moulding sowie die virtuelle Abbildung der gesamten Prozesskette bie- ten dabei ein hohes Transferpotenzial in die Automobilindustrie", erläutert Professor Dr. Peter Middendorf, Leiter des Instituts für Flugzeugbau. Gemeinsam mit Daimler wolle man zukünftig vor allem schnellere und kostengünstigere Verfahren untersuchen, um mit neuen Technologien den Herausforderungen einer Großserienproduktion zu begegnen.

Am Forschungscampus Arena-2036 (Active Research Environment for the Next Generation of Automobiles) sind neben sechs Instituten der Universität Stuttgart und Daimler zahlreiche weitere Vertreter aus Wirtschaft und Wissenschaft Baden-Württembergs be- teiligt. Der Campus wird vom Bundesministerium für Bildung und Forschung gefördert. Am Forschungscampus sollen Forscher aus der Wirtschaft und der Wissenschaft gemeinsam zu den Themen flexible Produktion und funktionsintegrierter Leichtbau forschen. Ziel des Konsortiums sei es, die produktorientierte Leichtbauentwicklung von Anfang an mit der Produktionsforschung zu verzahnen. Die Grundlagenforschung von Leichtbautechnologien und Produktionsmodellen sei auf das Auto der Zukunft im Jahr 2036, dem 150. Jubiläum des Automobils, ausgerichtet. 